\title{
Letter to the Editor: Spinopelvic Alignment and Low Back Pain after Total Hip Replacement Arthroplasty in Patients with Severe Hip Osteoarthritis
}

\author{
Mantu Jain ${ }^{1}$, Binod Kumar Behera ${ }^{2}$, Ayesha Mohapatara ${ }^{1}$ \\ ${ }^{I}$ Department of Orthopedics, All India Institute of Medical Sciences, Bhubaneswar, India \\ ${ }^{2}$ Department of Community Medicine and Family Medicine, All India Institute of Medical Sciences, Bhubaneswar, India
}

Dear Sir,

We have read the article entitled "Spinopelvic alignment and low back pain after total hip replacement arthroplasty in patients with severe hip osteoarthritis" by Eguchi et al. [1] with a lot of interest. First of all, we congratulate the authors for bringing out an important observation related to low back pain and spinopelvic parameters following total hip arthroplasty. However, we note some findings that we would share.

(1) The authors have used an unpaired t-test for various variables which they recorded before and after the hip arthroplasty, which we feel should have been a paired t-test. Since there is a change in the Visual Analog Scale (VAS) scores of hip pain and low back pain at three intervals, i.e., at preoperative, 1 month, and final follow-up, it would be interesting to reanalyze the data using the repeated measure analysis of variance (ANOVA). Finally, if required, a post hoc analysis can be applied after repeated measure ANOVA.

(2) Twelve patients with bilateral hip arthritis were operated by the authors. We believe they were operated unilaterally and not bilaterally. The VAS score in them for low back pain was not reduced as much as for unilateral arthritis. No mention about the coxalgia for the nonoperated side has been done at various time intervals for them.

(3) We also would like to know if the aetiology for total hip arthroplasty like ankylosing spondylitis (AS) and nonAS were evaluated in the author's study. Overall, the study is impressive and useful.

\section{Conflict of Interest}

No potential conflict of interest relevant to this article was reported.

\section{Author Contributions}

MJ, AM, and BKB have critically analyzed and prepared the article. All authors agree to the content of the final version.

Received April 12, 2019; Accepted April 13, 2019

Corresponding author: Mantu Jain

Department of Orthopedics, All India Institute of Medical Sciences, 106, Mahadev Orchid, Cosmopolis Road, Dumduma, Bhubaneswar, Odisha 751019, India

Tel: +91-0674-2473318, E-mail: mantu@aiimsbhubaneswar.edu.in 


\section{Reference}

1. Eguchi Y, Iida S, Suzuki C, et al. Spinopelvic alignment and low back pain after total hip replacement arthroplasty in patients with severe hip osteoarthritis. Asian Spine J 2018;12:325-34. 